\title{
A Deep Photometric Search for Substellar Mass Objects in Taurus
}

\author{
E. A. Magnier \\ Canada-France-Hawaii Telescope Corporation, 65-1238 Mamalahoa \\ Hwy, Kamuela, HI 96743 USA \\ C. Dougados, F. Ménard
}

Laboratoire d'Astrophysique de l'Observatoire de Grenoble, 414 rue de la Piscine 38041 Grenoble, France

E. L. Martín

Institute for Astronomy, University of Hawaii, 2680 Woodlawn Dr., Honolulu, HI 96822 USA

\author{
A. Magazzù \\ INAF-Centro Galileo Galilei, Apartado 565, 38700 Santa Cruz de La \\ Palma, Spain
}

\begin{abstract}
We have performed a photometric search for brown dwarf candidates in Taurus using the CFH12K wide-field mosaic camera. We are in the process of obtaining follow-up spectroscopy of likely brown dwarf candidates. We summarize the current state of the photometric analysis and the optical spectroscopy obtained to date. We also discuss the evidence for accretion in these systems.
\end{abstract}

\section{Introduction}

The formation and evolution of objects at or below the hydrogen burning limit is still poorly constrained. Since the first discoveries nearly a decade ago, a large number of old brown dwarfs have been identified (see for example these proceedings). These objects have ceased accretion and their evolution is determined by the internal details. Young brown dwarfs, with ages $<3 \mathrm{Myr}$, which are probably still accreting have only recently been identified in significant numbers (see Basri 2000 and references therein). Larger samples are still needed to explore issues such as the evolution of accretion and the initial mass function.

The Taurus star-forming region represents one of the most attractive sites to search for young brown dwarfs. Its advantages include the modest distance $(140 \mathrm{pc})$, the large areas with minimal extinction, and the appropriate level of stellar density - sufficiently high to provide large numbers of stars without excessive crowding. With these goals in mind, our team has performed a large optical survey of Taurus using the CFH12K wide-field mosaic imager. 
For a cluster with the age of Taurus $(\sim 4 \mathrm{Myr})$, the substellar limit corresponds to a spectral type of roughly M7V. Earlier work on Taurus (Briceño et al., 1998; Luhman 2000) had found no objects later than M6.5V, and implied that the fraction of brown dwarfs in Taurus may be low compared with other young clusters. However, with a total of only $0.7 \mathrm{deg}^{2}$, these earlier surveys did not cover a substantial fraction of the Taurus cloud. The large field-of-view of the CFH12K imager allowed us to significantly improve upon these earlier works.

\section{The Survey}

Our survey had two initial goals: to find brown dwarf candidates on the basis of their photometric properties, and to obtain spectra to identify any objects later than M7V. The longer-term goals include obtaining a sufficiently complete sample to place strong constraints on the Taurus IMF and to search for evidence of accretion in the brown dwarfs identified.

The CFH12K Taurus Survey consists of R, I, z', and $\mathrm{H} \alpha$ images of 11 Taurus fields with completeness limits of roughly 22.5, 21.5, and 22.0 for the three broad-band filters respectively (Dougados et al 2002). The images were processed using the CFHT analysis system, Elixir (Magnier \& Cuillandre 2002), and are photometrically calibrated using the well-determined zero-points for this camera. Photometric accuracy is roughly $2 \%$ per filter for the broad-band filters, and roughly $10 \%$ for the $\mathrm{H} \alpha$ filter.

The images were obtained in several batches and analysed in two main stages. The largest group of images was obtained during 4 photometric nights in Dec 1999 and Jan 2000 using Director's Discretionary time. These images were analysed as a single batch and initial follow-up projects carried out on the basis of this data set. The remainder of the images, including all z' images, as well as the last two fields, were obtained with a smattering of time in both PI and QSO modes over the period from Nov 2000 to Dec 2001. These images were analysed as a second batch and are now being examined in detail.

\section{The Candidates}

To date, we have had 3 major attempts at spectroscopic classification of our Taurus brown dwarf candidates. In each of these cases, a slightly different photometrically-selected sample was targeted. We adjusted the samples to match the capability of the telescopes used and to improve the selection based on additional information obtained.

Our initial photometric selection used the I, R-I color-magnitude diagram to identify objects with the appropriate colors and magnitudes. Lacking the 2MASS data at that time, and selecting targets for the $4.2 \mathrm{~m}$ William Herschel Telescope, we extracted objects which fell more than 2 magnitudes above the zero-age main sequence (ZAMS) for Taurus in the I, R-I diagram with $\mathrm{I}<18$. This initial selection yielded 31 candidates for which we obtained spectroscopy. This first spectroscopic follow-up study yielded 4 very strong candidates with spectral types later than $\mathrm{M} 7 \mathrm{~V}$ and with $\mathrm{H} \alpha$ emission. The presence of $\mathrm{H} \alpha$ emission and weak $\mathrm{NaI}$ absorption lines provide strong confirmation that the 


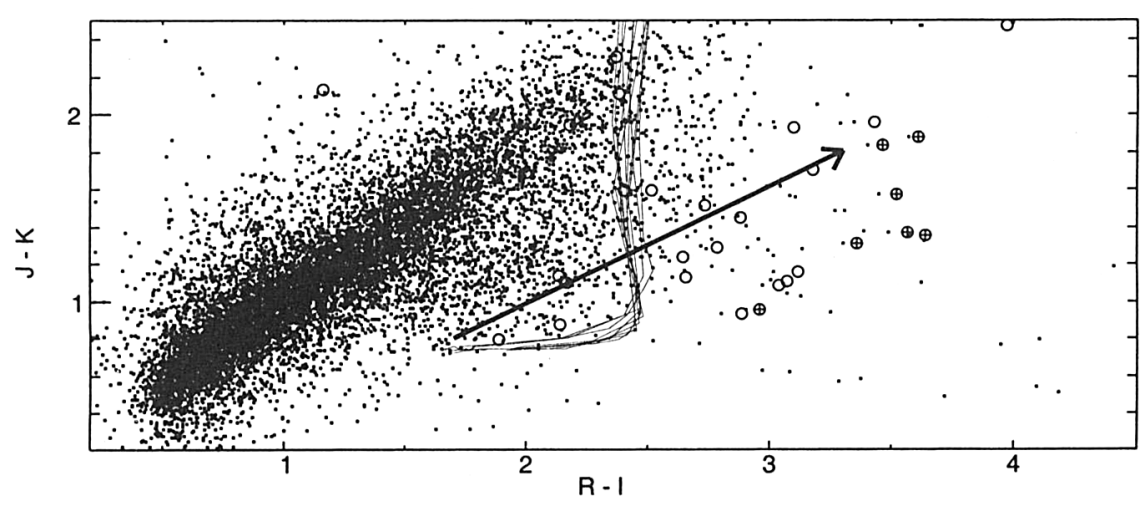

Figure 1. Taurus color-color diagram showing objects with spectroscopy (circles) and the best brown-dwarf candidates (crosses). Tracks from Chabrier (2000).

object of interest is in fact a young brown dwarf in Taurus, not a background giant with late spectral type. At this stage, our goal was to demonstrate the unambiguous presence of brown dwarfs in Taurus; while an object with strong $\mathrm{H} \alpha$ emission is very likely to be a Taurus brown dwarf, absence of $\mathrm{H} \alpha$ does not exciude the object as a brown dwarf. The four brown dwarfs found in these spectroscopic observations have been presented in detail elsewhere (Martín et al. 2001).

The second sample of objects was observed at the Keck $10 \mathrm{~m}$ telescope, so it was possible to explore the fainter areas of the color-magnitude diagram. We used essentially the same criteria as above, but relaxed the brightness to I $<20.1$, obtaining spectra of an additional 9 candidates. Among these candidates, nearly all showed cool spectral types, later than M4, in contrast with the brighter sample which was highly contaminated by reddened background objects. Of these objects, 1 shows a spectral type of approximate M7, and is therefore a brown-dwarf candidate, though the resolution is too poor to constrain the presence of $\mathrm{H} \alpha$. A second object has spectral type of roughly M6, and is therefore very close to the substellar boundary.

The third sample of objects was chosen for spectroscopy at the VLT. In an attempt to improve on the efficiency and reduce the number of background objects, we included the newly acquired 2MASS photometry in the sample selection. We chose objects 2 magnitude above the ZAMS in the I, R-I colormagnitude diagram, with $\mathrm{I}<20$, with the additional constraint that the object have I-J, J-K colors consistent with those of stars with spectral type M6 or later. At the time of this selection, only the first phase of optical observations had been performed and reduced. The selection resulted in a total of 26 new objects, 9 of which have had spectra obtained at the VLT with FORS. Of these candidates, nearly all are cool, with spectral types M4 or later, and 2 appear to have spectral types of $\mathrm{M} 7$ or later. One of these exhibits strong $\mathrm{H} \alpha$ emission. 
Now that all of our photometric data have been obtained and analysed, our next spectroscopic campaign, starting in the fall of 2002, will include targets from a substantially larger list of candidates. We can examine the I-J, J-K color-color diagram (Figure 1) to make predictions about the total number of brown dwarfs we will eventually identify. The region in this color-color diagram which contains all 7 of our likely brown dwarfs includes 16 objects for which we have spectroscopy. This same region also includes a total of 80 objects. If the detection efficiency for the remaining objects is the same as for the objects already observed, we should expect in the vicinity of $35 \pm 10$ brown dwarfs in our entire field.

\section{Evidence of Accretion}

We have been searching for evidence of accretion in several of the strongest candidate brown dwarfs. We have obtained HiRes echelle spectra of three of the brown dwarfs identified in Martín et al (2001). Two of these spectra show the $\mathrm{H} \alpha$ profiles expected for an accreting system: broad wings (FWZM $\sim 100$ - $200 \mathrm{~km} \mathrm{sec}^{-1}$ ), with blueshifted asymmetry. These high-resolution spectra suggest that the $\mathrm{H} \alpha$ emission in these systems is due to active accretion, not simply to chromospheric activity. Further evidence of accretion in objects near the substellar limit comes from the low mass star IRAS $04158+2805$, which we discuss in detail in Ménard et al. (2002). In this system, a substantial circumstellar disk is seen in silhouette and in reflection, and emission from a collimated outflow is also clearly seen.

\section{Conclusions}

The CFH12K Taurus Survey has successfully revealed brown dwarfs in this nearby center of star formation activity. Ongoing spectroscopic classification of the numerous candidates has revealed a total of 7 likely brown dwarfs and a large number of other cool objects just above the substellar limit. Our candidate selection criteria are becoming more efficient at revealing the coolest objects. Extrapolation of the observed objects implies a total of 25 - 45 brown dwarfs yet to be identified in our fields.

\section{References}

Basri, G. 2000, ARA\&A, 38, 485

Briceño, C., Hartmann, L., Stauffer, J., \& Martín, E. 1998, AJ, 115, 2074

Chabrier, G., Baraffe, I., Allard, F., \& Hauschildt, P 2000, AJ, 542, 464

Dougados, C., et al. 2002 in prep

Luhman, K. 2000, ApJ, 544, 1044

Magnier, E. \& Cuillandre, J.-C. 2002 in prep

Martín, E. L., Dougados, C., Magnier, E., Ménard, F., Magazzù, A., Cuillandre, J.-C., \& Delfosse, X 2001, ApJ, 561, 195

Ménard, F., Dougados, C., Magnier, E., et al., 2002 in prep 